\title{
Roxadustat for the Maintenance Treatment of Anemia in Patients with End-Stage Kidney Disease on Stable Dialysis: A European Phase 3, Randomized, Open-Label, Active-Controlled Study (PYRENEES)
}

\author{
Botond Csiky · Michael Schömig • Ciro Esposito · Jonathan Barratt • \\ Michael Reusch · Udaya Valluri · Wladyslaw Sulowicz
}

Received: June 24, 2021 / Accepted: August 26, 2021 / Published online: September 19, 2021

(C) The Author(s) 2021

\section{ABSTRACT}

Introduction: Roxadustat is an orally administered hypoxia-inducible factor prolyl hydroxylase inhibitor being developed for the treatment of anemia of chronic kidney disease (CKD). This European, phase 3, randomized, open-label, active-controlled study investigated efficacy

Supplementary Information The online version contains supplementary material available at https:// doi.org/10.1007/s12325-021-01904-6.

\section{B. Csiky $(\square)$}

2nd Department of Medicine and Nephrology-

Diabetes Center, University of Pécs, FMC Dialysis

Centers, Pécs, Hungary

e-mail: botond.csiky@gmail.com

M. Schömig

Dialysis Center, Heilbronn, Germany

C. Esposito

Unit of Nephrology and Dialysis, ICS Maugeri,

University of Pavia, Pavia, Italy

J. Barratt

University of Leicester, Leicester, UK

M. Reusch

Astellas Pharma Europe B.V., Leiden,

The Netherlands

U. Valluri

Astellas Pharma, Inc., Northbrook, IL, USA

W. Sulowicz

Department of Nephrology, Collegium Medicum of the Jagiellonian University, Krakow, Poland and safety of roxadustat in patients with endstage kidney disease on dialysis for at least 4 months.

Methods: Patients were randomized to switch from an erythropoiesis-stimulating agent (ESA) (epoetin alfa or darbepoetin alfa) to roxadustat three times/week or to continue their previous ESA. Roxadustat and ESA doses were adjusted to maintain hemoglobin within 10.0-12.0 g/dL during the treatment period (day 1 up to 52-104 weeks). Primary endpoints were hemoglobin change from baseline (CFB) to the average of weeks 28-36 without rescue therapy and hemoglobin CFB to the average of weeks $28-52$ regardless of rescue therapy. Treatment-emergent adverse events (TEAEs) were assessed descriptively.

Results: Of 1081 screened patients, 836 were randomized and received treatment (roxadustat, $n=415$; ESA, $n=421$ ). The least squares means $(95 \% \mathrm{CI})$ of the treatment difference (roxadustat - ESA) for hemoglobin CFB to weeks 28-36 (without rescue therapy) and CFB to weeks 28-52 (regardless of rescue therapy) were $0.235(0.132,0.339) \mathrm{g} / \mathrm{dL}$ and $0.171(0.082$, $0.261) \mathrm{g} / \mathrm{dL}$, respectively, demonstrating noninferiority of roxadustat to ESA (non-inferiority margin of $-0.75 \mathrm{~g} / \mathrm{dL}$ ). The proportions of patients who achieved target hemoglobin without rescue therapy during weeks 28-36 were $84.2 \%$ (roxadustat) and $82.4 \%$ (ESA). Roxadustat was superior to ESA in decreasing LDL cholesterol from baseline to the average of 
weeks 12-28. Serious TEAEs occurred in 50.7\% (roxadustat) and $45.0 \%$ (ESA) of patients. Common TEAEs in both treatment groups included hypertension, arteriovenous fistula thrombosis, headache, and diarrhea.

Conclusion: Roxadustat was non-inferior to ESAs in maintaining hemoglobin levels in this cohort of patients with anemia of CKD on dialysis for at least 4 months who were previously treated with ESAs. Observed TEAEs were consistent with previous studies.

Keywords: Hemodialysis; Peritoneal dialysis; Chronic kidney disease

\section{Key Summary Points}

This European, phase 3, randomized, open-label, active-controlled study investigated the efficacy and safety of roxadustat in patients with end-stage kidney disease on stable (prevalent) dialysis for at least 4 months.

Patients were randomized to (a) switch from their previous erythropoiesisstimulating agent (ESA) treatment (epoetin or darbepoetin alfa) to oral roxadustat therapy three times/week, or (b) to continue with their previous ESA.

The least squares means (95\% CI) of the treatment difference (roxadustat - ESA) for hemoglobin change from baseline to weeks 28-36 (without rescue therapy) and from baseline to weeks 28-52 (regardless of rescue therapy) were $0.235(0.132$, $0.339) \mathrm{g} / \mathrm{dL}$ and $0.171(0.082,0.261) \mathrm{g} / \mathrm{dL}$, respectively, demonstrating noninferiority of roxadustat to ESA (noninferiority margin of $-0.75 \mathrm{~g} / \mathrm{dL}$ ).

Roxadustat was also superior to ESA in decreasing LDL cholesterol from baseline to the average of weeks 12-28.

The most common treatment-emergent adverse events in both treatment groups were hypertension, arteriovenous fistula thrombosis, headache, and diarrhea.

\section{INTRODUCTION}

Anemia of dialysis-dependent (DD) CKD is associated with reduced quality of life and increased risk of hospitalization and mortality $[1,2]$. Anemia of DD CKD is commonly treated with erythropoiesis-stimulating agents (ESAs), alongside oral or intravenous (IV) iron supplementation; however, approximately $12 \%$ of patients with anemia of DD CKD do not respond adequately to ESAs [3, 4], thereby necessitating the use of higher doses to achieve the recommended hemoglobin ( $\mathrm{Hb})$ target $[5,6]$. Several analyses have suggested that higher ESA doses were associated with increased cardiovascular risk in some patients with CKD [7-9].

Roxadustat is an orally administered hypoxia-inducible factor (HIF) prolyl hydroxylase inhibitor in development for the treatment of anemia of CKD. HIF is an oxygen-sensitive transcription factor that regulates erythropoiesis by increasing endogenous erythropoietin production to near physiologic levels, decreasing hepcidin, thus promoting iron absorption and mobilization. In the presence of normal oxygen levels, HIF prolyl hydroxylase enzyme activity results in HIF- $\alpha$ degradation. During hypoxia, the enzyme is inactive, allowing the dimerization of HIF- $\alpha$ with HIF- $\beta$, leading to increased expression of erythropoietin, transferrin, and transferrin receptor, as well as increased iron absorption and mobilization from the reticuloendothelial systems (spleen, liver, and bone marrow). Similar to epoetin alfa and darbepoetin alfa (DA), HIF prolyl hydroxylase inhibitors stimulate an erythropoietic response by increasing erythropoietin. In addition, HIF prolyl hydroxylase inhibitors regulate iron metabolism and stimulate transcription of genes involved in the body's natural response to hypoxia in the presence of normal cellular oxygen levels $[1,10]$.

Roxadustat is approved in Chile, China, the European Union (EU), Japan, and South Korea for the treatment of patients with anemia in DD and non-dialysis-dependent (NDD) CKD. Global phase 3 studies have demonstrated the efficacy and safety of roxadustat in achieving and 
maintaining $\mathrm{Hb}$ target levels in patients with DD [11-13] and NDD [14, 15] CKD (NCT02964936; $\quad$ EudraCT numbers 2012-005180-27 and 2013-000951-42).

This phase 3 study evaluated the efficacy and safety of roxadustat compared with ESA (epoetin alfa or DA) in the maintenance treatment of anemia in patients with end-stage kidney disease (ESKD) on stable (prevalent) dialysis for at least 4 months who were receiving stable doses of either epoetin alfa or DA for the treatment of anemia. Since this is the first large study of roxadustat conducted in a mainly European population on dialysis for at least 4 months, its findings can contribute to an understanding of the consistency of response to roxadustat across CKD progression and in various geographic regions.

\section{METHODS}

\section{Study Design}

This was a phase 3 , multicenter, randomized, open-label, active-controlled study (EudraCT number 2013-001497-16) conducted at approximately 150 sites in 17 countries, mostly in Europe, between November 2014 and July 2018. After a screening period (at most 6 weeks), eligible patients were randomized (1:1) on day 1 of the treatment period to switch from their previous ESA treatment (any short-acting epoetin or DA) to oral roxadustat therapy (roxadustat group) or to continue with their previous IV or subcutaneous (SC) treatment with DA or epoetin alfa (i.e., switch to epoetin alfa if they were previously treated with any short-acting epoetin) at approximately the same average weekly dose and per the same route used prior to randomization (ESA group). Treatment started on day 1 and lasted from 52 weeks for up to 104 weeks. During a 4-week follow-up period, the choice of anemia treatment was at the discretion of the investigator (Fig. 1). Patients who prematurely discontinued study treatment completed the end of treatment (EOT) visits (EOT visit and EOT +2 weeks visit) and end of study (EOS) visit. Thereafter, patients who took at least one dose of study

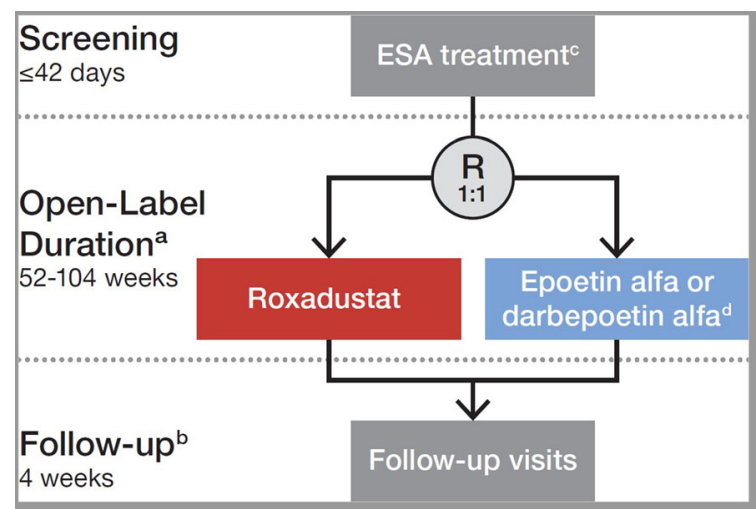

Fig. 1 Study flow chart. ${ }^{\mathrm{a}}$ Hemoglobin was maintained within $10-12 \mathrm{~g} / \mathrm{dL}$. ${ }^{\mathrm{b}}$ The choice of anemia treatment was at the discretion of the investigator. ${ }^{c}$ Patients could have taken epoetin (i.e., epoetin alfa, beta, theta, zeta, delta, or omega) or darbepoetin alfa prior to randomization.

${ }^{\mathrm{d}}$ Patients received epoetin alfa if they were on epoetin (i.e., epoetin alfa, beta, theta, zeta, delta, or omega) prior to randomization or darbepoetin alfa if they were on darbepoetin alfa prior to randomization. ESA erythropoiesis-stimulating agent, $R$ randomization

treatment were followed-up every 6 months for vital status, serious adverse events (SAEs), and cardiovascular and thromboembolic adverse events until their projected date of completion of the follow-up period (i.e., projected EOS visit) or until consent was withdrawn.

Two amendments were introduced in the study protocol to align with the global clinical development program of roxadustat (Supplemental Methods).

This study was conducted in accordance with the ethical principles of the Declaration of Helsinki, Good Clinical Practice, the International Committee on Harmonisation guidelines, and applicable laws and regulations. The protocol was approved by each institutional review board and all subjects provided written informed consent.

\section{Study Population}

Patients were aged at least 18 years; on hemodialysis (HD) or peritoneal dialysis (PD) for at least 4 months; had been treated with stable doses of epoetin or DA for at least 8 weeks; had mean $\mathrm{Hb}$ values within 9.5-12.0 g/ 
$\mathrm{dL}$, with an absolute difference no greater than $1.3 \mathrm{~g} / \mathrm{dL}$ between the highest and the lowest value during the screening period; and were iron replete (ferritin $\geq 100 \mathrm{ng} / \mathrm{mL}$ and transferrin saturation $[\mathrm{TSAT}] \geq 20 \%$ at screening). The full list of inclusion and exclusion criteria is reported in the Supplemental Methods.

\section{Study Drug Administration}

A block randomization schedule was used to randomize patients via an interactive response system (IRS) to receive roxadustat or ESA (epoetin alfa or DA) according to five stratification factors (previous ESA treatment [epoetin versus DA]; geographic region; history of cardiovascular, cerebrovascular, or thromboembolic diseases; average weekly ESA dose prior to randomization; and $\mathrm{Hb}$ value at screening). Each participating site was given the randomization number that was available in sequential order through the IRS. Patients were then enrolled by the investigators of each participating site. Assignment to treatment group was also done by the investigators, according to the aforementioned randomization allocation scheme. Roxadustat was supplied as 20-mg, 50-mg, and 100-mg tablets for oral administration three times weekly (TIW). The initial dose of roxadustat was based on the patient's average weekly dose of epoetin or DA during the 4 weeks prior to randomization in accordance with predefined rules (Supplemental Table S1). In general, dose adjustments for roxadustat were permitted to maintain $\mathrm{Hb}$ in the range of 10-12 g/dL every 4 weeks, starting from week 4 , and were conducted in accordance with prespecified rules (Supplemental Tables S2 and S3).

Epoetin alfa and DA were provided, as a solution, for SC or IV injection. The initial dose and frequency of ESA administration were at the investigator's discretion, provided that the average weekly dose remained approximately the same as the one prior to randomization. Dose adjustments to maintain $\mathrm{Hb}$ in the range of $10-12 \mathrm{~g} / \mathrm{dL}$ were permitted and were conducted in accordance with the European Union (EU) or UK Summary of Product Characteristics of DA and epoetin alfa, respectively.

\section{Endpoints and Assessments}

\section{Primary Efficacy Endpoints}

This study included two primary efficacy endpoints, each based on data supporting the submission to either EU or United States (US) health authorities (European Medicines Agency [EMA] and Food and Drug Administration [FDA], respectively) for the approval of roxadustat for the treatment of anemia in patients with ESKD on dialysis. The EU (EMA) primary endpoint was change in $\mathrm{Hb}$ levels from baseline to the average of weeks $28-36$, without the patient having received rescue therapy (i.e., red blood cell [RBC] transfusion for all patients and ESA treatment for those receiving roxadustat) within 6 weeks prior to and during weeks 28-36. The US (FDA) primary efficacy endpoint was change in $\mathrm{Hb}$ levels from baseline to the average of weeks $28-52$, regardless of rescue therapy.

\section{Secondary Efficacy and Safety Endpoints}

Key secondary endpoints are summarized in Table 1. Additional information is reported in the Supplemental Methods. Additional secondary endpoints are summarized in Table 2. Safety was assessed by monitoring treatmentemergent adverse events (TEAEs), vital signs, clinical laboratory results, and 12-lead electrocardiograms and reported for up to 28 days after last dose of study drug. The schedule of visits and assessments is provided in Supplemental Table S5.

\section{Statistical Methods}

This study was sufficiently powered for both primary efficacy endpoints (Supplemental Methods). The full analysis set (FAS) consisted of all randomized subjects who received at least one dose of study drug and had at least one post-dose $\mathrm{Hb}$ assessment; the per protocol set (PPS) included all FAS subjects who did not meet any of the PPS exclusion criteria; the safety analysis set (SAF) included all randomized patients who received at least one dose of study drug.

The primary EU (EMA) efficacy endpoint was analyzed using a mixed model of repeated 
Table 1 Key secondary efficacy endpoints

\begin{tabular}{|c|c|c|c|}
\hline $\begin{array}{l}\text { Test } \\
\text { sequence }\end{array}$ & Endpoint & $\begin{array}{l}\text { Analysis method } \\
\text { (analysis set) }\end{array}$ & Test type \\
\hline $\begin{array}{l}\text { Key } \\
\text { secondary } \\
1\end{array}$ & $\begin{array}{l}\mathrm{Hb} \text { response defined as proportion of patients achieving mean } \mathrm{Hb} \\
\text { levels } 10.0-12.0 \mathrm{~g} / \mathrm{dL} \text { during weeks } 28-36 \text { without having } \\
\text { received rescue therapy within } 6 \text { weeks prior to and during } \\
\text { weeks } 28-36\end{array}$ & $\begin{array}{l}\text { Miettinen and } \\
\text { Nurminen method CI } \\
\text { (PPS) }\end{array}$ & $\begin{array}{l}\text { Non- } \\
\text { inferiority }\end{array}$ \\
\hline $\begin{array}{l}\text { Key } \\
\text { secondary } \\
2\end{array}$ & $\begin{array}{l}\text { Change in low-density lipoprotein cholesterol from baseline to the } \\
\text { average of weeks } 12-28\end{array}$ & MMRM (FAS) & Superiority \\
\hline $\begin{array}{l}\text { Key } \\
\text { secondary } \\
3\end{array}$ & Mean monthly use of IV iron from day 1 to week 36 & ANCOVA (FAS) & Superiority \\
\hline $\begin{array}{l}\text { Key } \\
\text { secondary } \\
4\end{array}$ & $\begin{array}{l}\text { Change in SF-36 PF sub-score from baseline to the average of } \\
\text { weeks } 12-28^{a}\end{array}$ & MMRM (PPS) & $\begin{array}{l}\text { Non- } \\
\text { inferiority }\end{array}$ \\
\hline $\begin{array}{l}\text { Key } \\
\text { secondary } \\
5\end{array}$ & $\begin{array}{l}\text { Change in SF-36 VT sub-score from baseline to the average of } \\
\text { weeks } 12-28^{a}\end{array}$ & MMRM (PPS) & $\begin{array}{l}\text { Non- } \\
\text { inferiority }\end{array}$ \\
\hline $\begin{array}{l}\text { Key } \\
\text { secondary } \\
6\end{array}$ & Change in MAP ( $\mathrm{mmHg}$ ) from baseline to weeks $20-28$ & MMRM (PPS) & $\begin{array}{l}\text { Non- } \\
\text { inferiority }\end{array}$ \\
\hline $\begin{array}{l}\text { Key } \\
\text { secondary } \\
7\end{array}$ & Time to increase in blood pressure between weeks 1 and $36^{\mathrm{b}}$ & $\begin{array}{l}\text { Stratified Cox } \\
\text { proportional hazards } \\
\text { (PPS) }\end{array}$ & $\begin{array}{l}\text { Non- } \\
\text { inferiority }\end{array}$ \\
\hline $\begin{array}{l}\text { Key } \\
\text { secondary } \\
8\end{array}$ & Change in MAP ( $\mathrm{mmHg}$ ) from baseline to weeks $20-28$ & MMRM (FAS) & Superiority \\
\hline $\begin{array}{l}\text { Key } \\
\text { secondary } \\
9\end{array}$ & Time to increase in blood pressure between weeks 1 and 36 & $\begin{array}{l}\text { Stratified Cox } \\
\text { proportional hazards } \\
\text { (FAS) }\end{array}$ & Superiority \\
\hline
\end{tabular}

$C I$ confidence interval, $F A S$ full analysis set, $H b$ hemoglobin, $I V$ intravenous, $M A P$ mean arterial pressure, $M M R M$ mixed model of repeated measures method, PPS per protocol set, $S F-36$ PF Short Form-36 health survey physical functioning, $S F$ $36 V T$ Short Form-36 health survey Vitality, US United States

a Score range: 0-100; higher scores indicate better health status. US-normalized values were used for the analysis where the scores normed to the US population have a mean of 50 and standard deviation of 10

${ }^{b}$ Increase of at least $20 \mathrm{mmHg}$ systolic blood pressure (SBP) with SBP $\geq 170 \mathrm{mmHg}$ or increase of at least $15 \mathrm{mmHg}$ diastolic blood pressure (DBP) with DBP $\geq 100 \mathrm{mmHg}$

The FAS consisted of all randomized subjects who received at least one dose of study drug and had at least one post-dose $\mathrm{Hb}$ assessment; the PPS included all FAS subjects who did not meet any of the PPS exclusion criteria (Supplemental Table S4); the safety analysis set (SAF) included all randomized patients who received at least one dose of study drug 
Table 2 Additional secondary endpoints

\section{Endpoint}

1 Change from baseline to the average of weeks 12-28 in FACT-An

2 Change from baseline to the average of weeks 12-28 in EQ-5D-5L

3 Change from baseline to the average of weeks 12-28 in PGIC

4 Occurrence and duration of hospitalizations

5 Serum hepcidin

6 Serum ferritin

7 Transferrin saturation

EQ-5D-5L European Quality of Life 5-Dimension 5-Level, FACT-An Functional Assessment of Cancer TherapyAnemia, PGIC Patients' Global Impression of Change

measures method, adjusting for covariates (categorical variables: previous ESA treatment, region, history of cardiovascular disease, visits and visit by treatment; continuous variable: baseline $\mathrm{Hb}$ and baseline $\mathrm{Hb}$ by visit), to compare the roxadustat and ESA groups for the change of $\mathrm{Hb}$ level from baseline to weeks $28-36$ in the PPS. The null hypothesis was that the EU primary efficacy endpoint in the roxadustat arm was less than equal to the EU primary efficacy endpoint in the ESA arm minus $0.75 \mathrm{~g} / \mathrm{dL}$.

The primary US (FDA) efficacy endpoint was analyzed using an analysis of covariance model with multiple imputations, adjusting for covariates (categorical variables: previous ESA treatment, region, history of cardiovascular disease; continuous variable: baseline $\mathrm{Hb}$ ), to compare the roxadustat and ESA treatment groups for the change in $\mathrm{Hb}$ level from baseline to weeks 28-52, using all randomized patients. The null hypothesis was that the US (FDA) primary efficacy endpoint in the roxadustat arm was less than equal to the US (FDA) primary efficacy endpoint in the ESA arm minus $0.75 \mathrm{~g} /$ dL.

For all secondary endpoints, inferential analyses were used to evaluate the difference between roxadustat and ESA treatment groups. The key secondary endpoints were tested using a fixed-sequence testing procedure. If the null hypothesis is rejected for the EU (EMA) primary efficacy endpoint for both the total population and the subset population using Bonferronibased chain procedure, then the key secondary endpoints are tested for the total population. The subset population was defined as patients with an average prescribed weekly epoetin or darbepoetin dose within the last 4 weeks prior to randomization of at most $200 \mathrm{IU} / \mathrm{kg}$ or at most $1 \mu \mathrm{g} / \mathrm{kg}$. For each of the key secondary endpoints, if the null hypothesis is rejected for a test, the claim of superiority (or non-inferiority) is considered successful, and the test progresses to the next comparison in sequence. The PPS and the FAS were used for non-inferiority tests and superiority tests, respectively. Upon study completion, the prespecified subset population was 0.9757 (764/783), which led to a one-sided significance level of 0.02177 using Bonferronibased chain procedure. All data processing, summarization, and analyses were performed using SAS ${ }^{\circledR}$ Version 9.3.

\section{RESULTS}

\section{Patient Disposition}

Of 1081 patients who signed informed consent, 838 were randomized and 836 received treatment (roxadustat, $n=415$; ESA, $n=421$ [DA, $n=163$; epoetin alfa, $n=258]$ ) and were analyzed. The numbers of patients randomized after the first protocol amendment (May 13, 2015) in the roxadustat and ESA groups were 183 and 196, respectively. A total of 558 (roxadustat group, $n=249[60.0 \%]$; ESA group, $n=309$ [73.4\%]) patients completed 2 years of treatment, whereas $40.0 \%$ and $26.6 \%$ of patients discontinued treatment in the roxadustat group and ESA group, respectively (Fig. 2).

Demographics and baseline characteristics were generally comparable in the roxadustat and ESA (epoetin alfa and DA) treatment groups (Table 3). Over one-quarter $(28.4 \%)$ of patients had a history of diabetes (roxadustat $25.1 \%$ vs 
ESA 31.7\%). The majority of patients were randomized in Central and Eastern Europe (78.9\%). Most patients were receiving HD; however, the proportion of patients receiving PD was higher in the roxadustat group $(8.5 \%)$ versus the ESA group (3.6\%). The mean (SD) time on dialysis at baseline was 4.35 (4.18) (median 2.89; range $0.35-27.04)$ years in the roxadustat group and 4.09 (3.65) (median 2.97; range 0.33-20.86) years in the ESA group (Table 3 ). In the subgroup of patients previously treated with DA, the median time on dialysis at baseline was shorter in the roxadustat group (2.75 years; range $0.38-20.88$ years) versus the DA group (3.41 years; range $0.33-20.86$ years), whereas in the subgroup previously treated with epoetin alfa, time on dialysis was longer in the roxadustat group (3.14 years; range 0.3527.04 years) than in the epoetin alfa group (2.60 years; range $0.34-17.36$ years) (Supplemental Table S6).

There appeared to be imbalances between the roxadustat and ESA groups in comorbidities. The proportion of patients with a history of cardiovascular, cerebrovascular, or thromboembolic diseases (40.8\% vs $47.9 \%)$; type 2 diabetes mellitus (21.5\% vs $30.2 \%$ ); or history of diabetes $(25.1 \%$ vs $31.7 \%)$ was lower in the roxadustat group than in the ESA group (Table 3). There also appeared to be imbalances between the roxadustat subgroups and the subgroups previously treated with epoetin alfa and with DA (Supplemental Table 6). In the subgroup previously treated with epoetin alfa, the proportion of patients with history of diabetes was lower in the roxadustat subgroup $(23.8 \%)$ than in the epoetin alfa group (31.5\%). Additionally, dialysis vintage was shorter in the roxadustat subgroup compared to the DA subgroup (3.89 vs 4.76 years) but longer compared to the epoetin alfa subgroup (4.63 vs 3.67 years).

\section{Treatment Exposure and Compliance}

The median treatment duration during the study was comparable between the treatment groups (roxadustat 103.71 [range 0.1-104.7] weeks; ESA 103.14 [range 0.1-104.4] weeks); mean treatment duration was lower in the roxadustat treatment group (80.30 weeks) compared with the ESA treatment group (89.42 weeks). Patient exposure years (PEY) on treatment in the study were 637.2 years in the roxadustat group and 719.7 years in the ESA group. The mean (SD) weekly dose prescribed over weeks 1-4 was 315 (75.9) mg for roxadustat, 6241.2 (4184.0) IU for epoetin alfa, and 26.3 (15.8) $\mu \mathrm{g}$ for DA. The mean (SD) weekly dose by weight prescribed over weeks $1-4$ was 4.3 (1.2) $\mathrm{mg} / \mathrm{kg}$ for roxadustat, $85.8(58.8) \mathrm{IU} / \mathrm{kg}$ for epoetin alfa, and $0.3(0.2) \mu \mathrm{g} / \mathrm{kg}$ for DA. The mean (SD) weekly dose administered during the treatment period (day 1-week 104) was 252.3 (133.0) $\mathrm{mg}$ for roxadustat, 7598.3 (4787.4) IU for epoetin alfa, and 32.3 (23.8) $\mu \mathrm{g}$ for DA (Supplemental Figs. S1, S2, and S3). Treatment compliance (defined as the dispensed amount minus the returned amount relative to the prescribed dose $\times 100$ ) was comparable in the roxadustat and ESA treatment groups (100.2\% vs $99.1 \%)$.

\section{Primary Efficacy Endpoint (EU [EMA])}

In the PPS, the least squares mean (LSM; 95\% CI) $\mathrm{Hb}$ change from baseline to weeks 28-36 was $0.428(0.350,0.506) \mathrm{g} / \mathrm{dL}$ in the roxadustat group and $0.193(0.117,0.268) \mathrm{g} / \mathrm{dL}$ in the ESA group. The LSM $(95 \% \mathrm{CI})$ of the difference between roxadustat and ESA was $0.235(0.132$, $0.339) \mathrm{g} / \mathrm{dL}$, and since the lower bound of the $95 \%$ CI was higher than $-0.75 \mathrm{~g} / \mathrm{dL}$, non-inferiority of roxadustat versus ESA was declared for the change in $\mathrm{Hb}$ levels from baseline to the average of weeks 28-36 (without rescue therapy) (Table 4). Upon study completion, the prespecified subset population was 0.9757 (764/ 783 ), which leads to a one-sided significance level of 0.02177 using Bonferroni-based chain procedure. The $P$ values for testing both the total study population and the subset (results are not presented for subset) were both below 0.001 , indicating noninferiority of roxadustat in both populations. Similar results were found in the subgroups of patients previously treated with DA or epoetin (Supplemental Table S7). A consistent treatment effect was shown across all subgroup analyses, defined by the categorical 


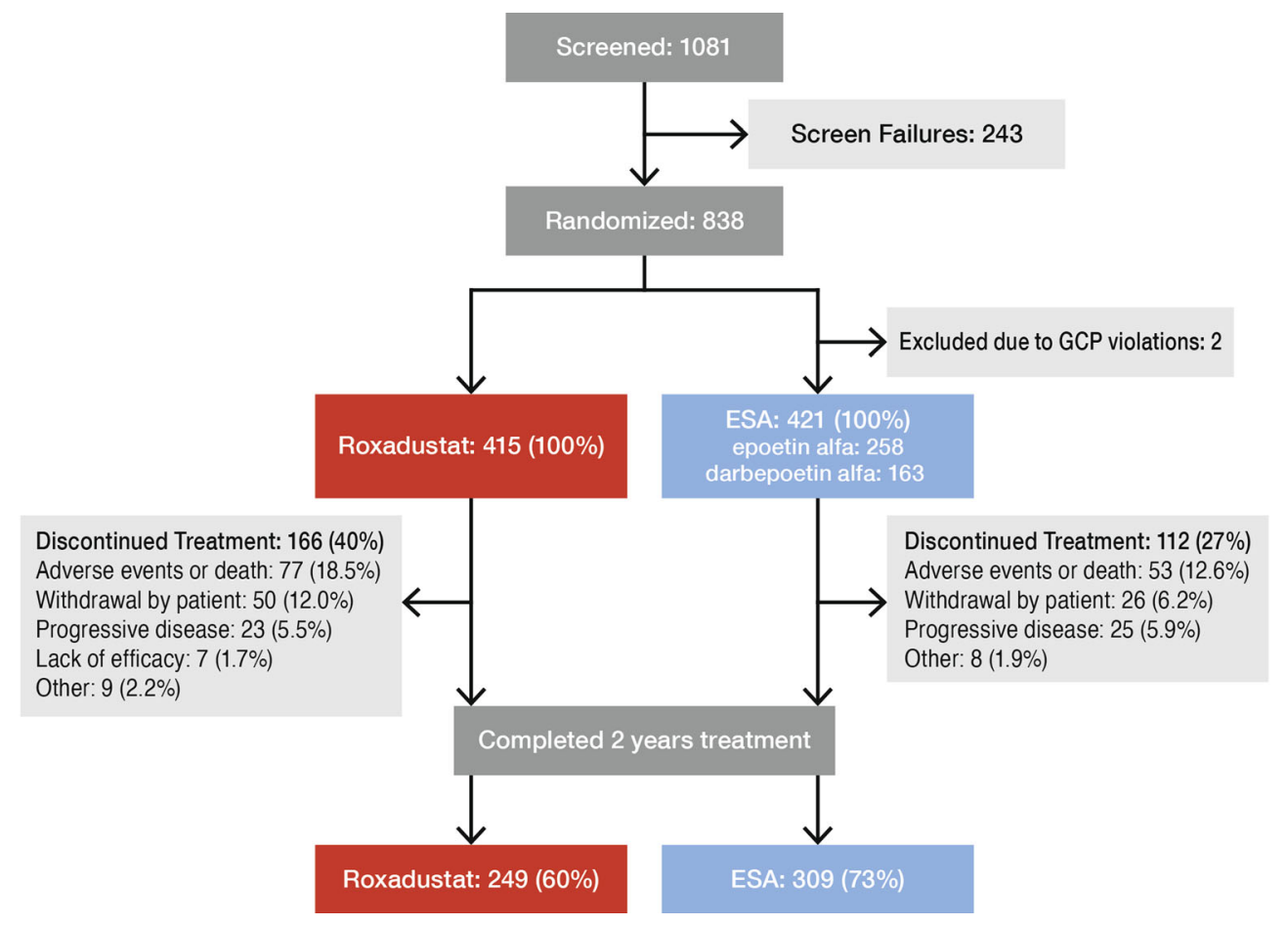

Fig. 2 Patient disposition. ESA erythropoiesis-stimulating agent, $G C P$ good clinical practices

variables of age; sex; region; baseline $\mathrm{Hb}$; dialysis type; history of cardiovascular, cerebrovascular, or thromboembolic diseases; and baseline high-sensitivity C-reactive protein.

Mean $\mathrm{Hb}$ concentrations from baseline to week 52 and changes from baseline at each visit are shown in Fig. 3 and Supplemental Fig. S4, respectively.

\section{Primary Efficacy Endpoint (US [FDA])}

In all randomized patients, the baseline (SD) $\mathrm{Hb}$ levels were comparable between the roxadustat and ESA treatment groups. The LSM (95\% CI) $\mathrm{Hb}$ change from baseline to weeks $28-52$ was $0.363(0.288,0.438) \mathrm{g} / \mathrm{dL}$ in the roxadustat group and $0.192(0.121,0.262) \mathrm{g} / \mathrm{dL}$ in the ESA group. The LSM of the difference between roxadustat and ESA (95\% CI) was 0.171 (0.082, $0.261) \mathrm{g} / \mathrm{dL}$, and since the lower bound of the 95\% CI was higher than $-0.75 \mathrm{~g} / \mathrm{dL}$, non-inferiority of roxadustat versus ESA was declared for the change in $\mathrm{Hb}$ levels from baseline to the average of weeks 28-52 (regardless of rescue therapy) (Table 5).
The same finding was confirmed in the subgroups of patients previously treated with DA or epoetin (Supplemental Table S8).

In the FAS, the use of rescue therapy was required in $44(10.7 \%$; RBC transfusions, 38 [9.2\%]; ESA, 6 [1.5\%]) and 54 (12.9\%; RBC transfusion) patients in the roxadustat and ESA treatment groups, respectively. In the FAS, IV iron was used in 104 (25.2\%) and 235 (56.0\%) patients in the roxadustat and ESA groups, respectively, during the efficacy-emergent period (from first drug dose to EOT visit), with a mean (SD) monthly dose of 12.0 (47.6) mg (roxadustat) and 44.8 (88.6) $\mathrm{mg}$ (ESA) from day 1 through week 36 (Supplemental Fig. S5).

\section{Key Secondary Efficacy Endpoints}

Results for the key secondary endpoints are summarized in Table 6 . The sequential analyses of the key secondary endpoints demonstrated superiority of roxadustat versus ESA for change in LDL cholesterol from baseline to the average of weeks 12-28 (difference of LSM - 0.377, 95\% CI -0.451 to $-0.304, p<0.001)$ and mean 
Table 3 Demographics and baseline characteristics (safety analysis set)

\begin{tabular}{|c|c|c|c|}
\hline Parameter & $\begin{array}{l}\text { Roxadustat } \\
(n=414)\end{array}$ & $\operatorname{ESA}(n=420)$ & Total $(N=834)$ \\
\hline Sex, male, $n(\%)$ & $245(59.2)$ & $235(56.0)$ & $480(57.6)$ \\
\hline \multicolumn{4}{|l|}{ Race, $n(\%)$} \\
\hline White & $405(97.8)$ & 407 (96.9) & $812(97.4)$ \\
\hline Black & $6(1.4)$ & $6(1.4)$ & $12(1.4)$ \\
\hline Asian & $1(0.2)$ & $3(0.7)$ & $4(0.5)$ \\
\hline Other & $2(0.5)$ & $4(1.0)$ & $6(0.7)$ \\
\hline Age, years & $61.0(13.8)$ & $61.8(13.4)$ & $61.4(13.6)$ \\
\hline Weight, kg & $76.29(15.88)$ & $76.18(17.25)$ & $76.23(16.58)$ \\
\hline BMI, $\mathrm{kg} / \mathrm{m}^{2}$ & $26.87(4.86)$ & $26.95(5.59)$ & $26.91(5.24)$ \\
\hline \multicolumn{4}{|l|}{ Region, $n$ (\%) } \\
\hline Western Europe & $86(20.8)$ & $90(21.4)$ & $176(21.1)$ \\
\hline Central and Eastern Europe & $328(79.2)$ & $330(78.6)$ & $658(78.9)$ \\
\hline \multicolumn{4}{|l|}{ Country, $n(\%)$} \\
\hline Bulgaria & $69(16.7)$ & $87(20.7)$ & $156(18.7)$ \\
\hline Hungary & $63(15.2)$ & $73(17.4)$ & $136(16.3)$ \\
\hline Russian Federation & $52(12.6)$ & $46(11.0)$ & $98(11.8)$ \\
\hline Serbia & $51(12.3)$ & $35(8.3)$ & $86(10.3)$ \\
\hline Croatia & $28(6.8)$ & $31(7.4)$ & $59(7.1)$ \\
\hline Romania & $20(4.8)$ & $20(4.8)$ & $40(4.8)$ \\
\hline Italy & $19(4.6)$ & $20(4.8)$ & $39(4.7)$ \\
\hline Poland & $18(4.3)$ & $11(2.6)$ & $29(3.5)$ \\
\hline Spain & $17(4.1)$ & $12(2.9)$ & $29(3.5)$ \\
\hline Germany & $15(3.6)$ & $19(4.5)$ & $34(4.1)$ \\
\hline Slovakia & $15(3.6)$ & $17(4.0)$ & $32(3.8)$ \\
\hline Portugal & $11(2.7)$ & $6(1.4)$ & $17(2.0)$ \\
\hline Belgium & $11(2.7)$ & $20(4.8)$ & $31(3.7)$ \\
\hline Other & $25(6.0)$ & $23(5.5)$ & $48(5.8)$ \\
\hline $\mathrm{Hb}, \mathrm{g} / \mathrm{dL}$ & $10.75(0.62)$ & $10.78(0.62)$ & $10.76(0.62)$ \\
\hline LDL cholesterol ${ }^{\mathrm{a}}, \mathrm{mmol} / \mathrm{L}$ & $2.750(1.017)$ & $2.644(1.015)$ & $2.697(1.017)$ \\
\hline \multicolumn{4}{|l|}{ Previous ESA treatment, $n(\%)$} \\
\hline Epoetin & $256(61.8)$ & $257(61.2)$ & $513(61.5)$ \\
\hline Darbepoetin alfa & $158(38.2)$ & $163(38.8)$ & $321(38.5)$ \\
\hline
\end{tabular}


Table 3 continued

\begin{tabular}{|c|c|c|c|}
\hline Parameter & $\begin{array}{l}\text { Roxadustat } \\
(n=414)\end{array}$ & ESA $(n=420)$ & Total $(N=834)$ \\
\hline \multicolumn{4}{|l|}{ Previous ESA dose/week ${ }^{\mathrm{b}}, n(\%)$} \\
\hline$\leq 200 \mathrm{IU} / \mathrm{kg}$ epoetin or $\leq 1 \mu \mathrm{g} / \mathrm{kg}$ darbepoetin alfa & $406(98.1)$ & $407(96.9)$ & $813(97.5)$ \\
\hline$>200 \mathrm{IU} / \mathrm{kg}$ epoetin or $>1 \mu \mathrm{g} / \mathrm{kg}$ darbepoetin alfa & $8(1.9)$ & $13(3.1)$ & $21(2.5)$ \\
\hline \multicolumn{4}{|l|}{ Previous ESA dose/week ${ }^{\mathrm{b}}, n(\%)$} \\
\hline$<25 \mu$ g darbepoetin alfa or $<5000$ IU epoetin & $222(53.6)$ & $189(45.0)$ & $411(49.3)$ \\
\hline $\begin{array}{l}25 \text { to }<40 \mu \mathrm{g} \text { darbepoetin alfa or } 5000 \text { to }<8000 \mathrm{IU} \\
\text { epoetin }\end{array}$ & $111(26.8)$ & $133(31.7)$ & $244(29.3)$ \\
\hline $\begin{array}{l}40 \text { to }<80 \mu \mathrm{g} \text { darbepoetin alfa or } 8000 \text { to }<16,000 \mathrm{IU} \\
\text { epoetin }\end{array}$ & $77(18.6)$ & $93(22.1)$ & $170(40.4)$ \\
\hline$\geq 80 \mu \mathrm{g}$ darbepoetin alfa or $\geq 16,000 \mathrm{IU}$ epoetin & $4(1.0)$ & $5(1.2)$ & $9(1.1)$ \\
\hline Time from CKD diagnosis, years & $8.81(7.08)$ & $8.29(6.65)$ & $8.55(6.87)$ \\
\hline \multicolumn{4}{|l|}{ CKD etiology, $n(\%)$} \\
\hline Hypertensive nephropathy & $124(30.0)$ & $120(28.6)$ & $244(29.3)$ \\
\hline Diabetic nephropathy & $74(17.9)$ & $95(22.6)$ & $169(20.3)$ \\
\hline Glomerulonephritis unspecified & $56(13.5)$ & $56(13.3)$ & $112(13.4)$ \\
\hline Pyelonephritis & $48(11.6)$ & $40(9.5)$ & $88(10.6)$ \\
\hline \multicolumn{4}{|l|}{ Baseline dialysis type, $n(\%)$} \\
\hline Hemodialysis & $379(91.5)$ & $405(96.4)$ & $784(94.0)$ \\
\hline Peritoneal dialysis & $35(8.5)$ & $15(3.6)$ & $50(6.0)$ \\
\hline \multicolumn{4}{|l|}{ Baseline CRP (nmol/L) } \\
\hline$\leq \mathrm{ULN}$ & $210(50.7)$ & $226(53.8)$ & $436(52.3)$ \\
\hline$>\mathrm{ULN}$ & $204(49.3)$ & $194(46.2)$ & $398(47.7)$ \\
\hline \multicolumn{4}{|l|}{ Dialysis vintage, years } \\
\hline Mean (SD) & $4.35(4.18)$ & $4.10(3.65)$ & $4.22(3.92)$ \\
\hline Median (min, max) & $2.89(0.35,27.04)$ & $2.97(0.33,20.86)$ & $2.95(0.33,27.04)$ \\
\hline \multicolumn{4}{|l|}{ Iron repletion at baseline, $n(\%)$} \\
\hline Ferritin $\geq 100 \mathrm{ng} / \mathrm{mL}$ and TSAT $\geq 20 \%$ & $355(86.0)$ & $366(87.1)$ & $721(86.6)$ \\
\hline Ferritin $<100 \mathrm{ng} / \mathrm{mL}$ or TSAT $<20 \%$ & $58(14.0)$ & $54(12.9)$ & $112(13.4)$ \\
\hline \multicolumn{4}{|l|}{ Blood pressure $^{\mathrm{a}}, \mathrm{mmHg}$} \\
\hline Systolic & $135.2(17.6)$ & $136.9(18.9)$ & $136.0(18.3)$ \\
\hline Diastolic & $75.2(11.0)$ & $74.3(11.2)$ & 74.8 (11.1) \\
\hline
\end{tabular}


Table 3 continued

\begin{tabular}{llll}
\hline Parameter & $\begin{array}{l}\text { Roxadustat } \\
(\boldsymbol{n}=\mathbf{4 1 4})\end{array}$ & ESA ( $\mathbf{n = 4 2 0 )}$ & Total $(\boldsymbol{N}=\mathbf{8 3 4})$ \\
\hline $\begin{array}{l}\text { History of cardiovascular, cerebrovascular, or } \\
\text { thromboembolic diseases }\end{array}$ & $169(40.8)$ & $201(47.9)$ & $370(44.4)$ \\
$\begin{array}{l}\text { Type 2 diabetes mellitus, } n(\%) \\
\text { History of diabetes, } n(\%)\end{array}$ & $89(21.5)$ & $127(30.2)$ & $216(25.9)$ \\
\hline
\end{tabular}

Data are reported as mean $(\mathrm{SD})$ unless otherwise indicated

$B M I$ body mass index, $C K D$ chronic kidney disease, ESA erythropoiesis-stimulating agent, $H b$ hemoglobin, $L D L$ lowdensity lipoprotein, $S D$ standard deviation, TSAT transferrin saturation, ULN upper limit of normal

${ }^{a}$ Data from all randomized patients

b Average weekly ESA dose in the 4 weeks prior to randomization

${ }^{c}$ Derived and based on selected preferred terms from the electronic clinical record form used for the analyses

Table 4 Statistical analysis of change in $\mathrm{Hb}$ levels from baseline to the average of weeks $28-36$ without rescue therapy (per protocol set)

\begin{tabular}{|c|c|c|}
\hline & $\begin{array}{l}\text { Roxadustat } \\
(n=386)\end{array}$ & $\begin{array}{l}\text { ESA } \\
(n=397)\end{array}$ \\
\hline Baseline $\mathrm{Hb}, \mathrm{g} / \mathrm{dL}$ & $\begin{array}{l}10.753 \\
(0.622)\end{array}$ & $\begin{array}{l}10.771 \\
\quad(0.628)\end{array}$ \\
\hline$N$ & 386 & 397 \\
\hline $\begin{array}{l}\text { Average Hb (weeks 28-36), } \\
\text { g/dL }\end{array}$ & $\begin{array}{l}11.231 \\
(0.667)\end{array}$ & $\begin{array}{l}10.979 \\
(0.777)\end{array}$ \\
\hline$N$ & 354 & 381 \\
\hline $\begin{array}{l}\mathrm{Hb} \text { change from BL to average } \\
\text { of weeks } 28-36\end{array}$ & $\begin{array}{l}0.477 \\
\quad(0.812)\end{array}$ & $\begin{array}{l}0.205 \\
\quad(0.941)\end{array}$ \\
\hline $\begin{array}{l}\text { LSM ( } 95 \% \mathrm{CI}) \text { change from } \\
\text { BL to average of } \\
\text { weeks } 28-36\end{array}$ & $\begin{array}{l}0.428 \\
(0.350 \\
0.506)\end{array}$ & $\begin{array}{l}0.193 \\
(0.117 \\
0.268)\end{array}$ \\
\hline $\begin{array}{l}\text { LSM difference } \\
\text { (roxadustat - ESA) }(95 \% \\
\text { CI) }\end{array}$ & \multicolumn{2}{|c|}{$0.235(0.132,0.339)$} \\
\hline $\begin{array}{l}\text { Two-sided } P \text { value (non- } \\
\text { inferiority) }\end{array}$ & \multicolumn{2}{|c|}{$<0.001$} \\
\hline
\end{tabular}

Data are reported as mean (standard deviation) unless otherwise indicated

$B L$ baseline, $C I$ confidence interval, ESA erythropoiesisstimulating agent, $H b$ hemoglobin, $L S M$ least squares mean monthly use of IV iron (day 1-week 36); noninferiority of roxadustat versus ESA was demonstrated for $\mathrm{Hb}$ response during weeks 28-36, change in Short Form-36 health survey Physical Functioning (SF-36 PF) and Short Form-36 health survey Vitality (SF-36 VT) sub-scores from baseline to the average of weeks 12-28, change in mean arterial pressure (MAP) from baseline to weeks 20-28, and time to increase in blood pressure between weeks 1 and 36. As part of the hierarchical testing, superiority was not demonstrated for roxadustat versus ESA for change in MAP from baseline to weeks $20-28$ or time to increase in blood pressure (week 1-week 36). The mean (SD) levels of LDL cholesterol over time are presented in Fig. 4.

\section{Additional Secondary Endpoints}

Changes from baseline to the average of weeks 12-28 in Functional Assessment of Cancer Therapy-Anemia (FACT-An) total score and EQ-5D-5L visual analogue scale (VAS), and the occurrence and duration of hospitalization, were comparable between treatment groups. A greater proportion of patients in the roxadustat versus the ESA group reported an improvement on the health-related quality of life (HRQoL) questionnaire Patients' Global Impression of Change (PGIC) (Supplemental Results). 


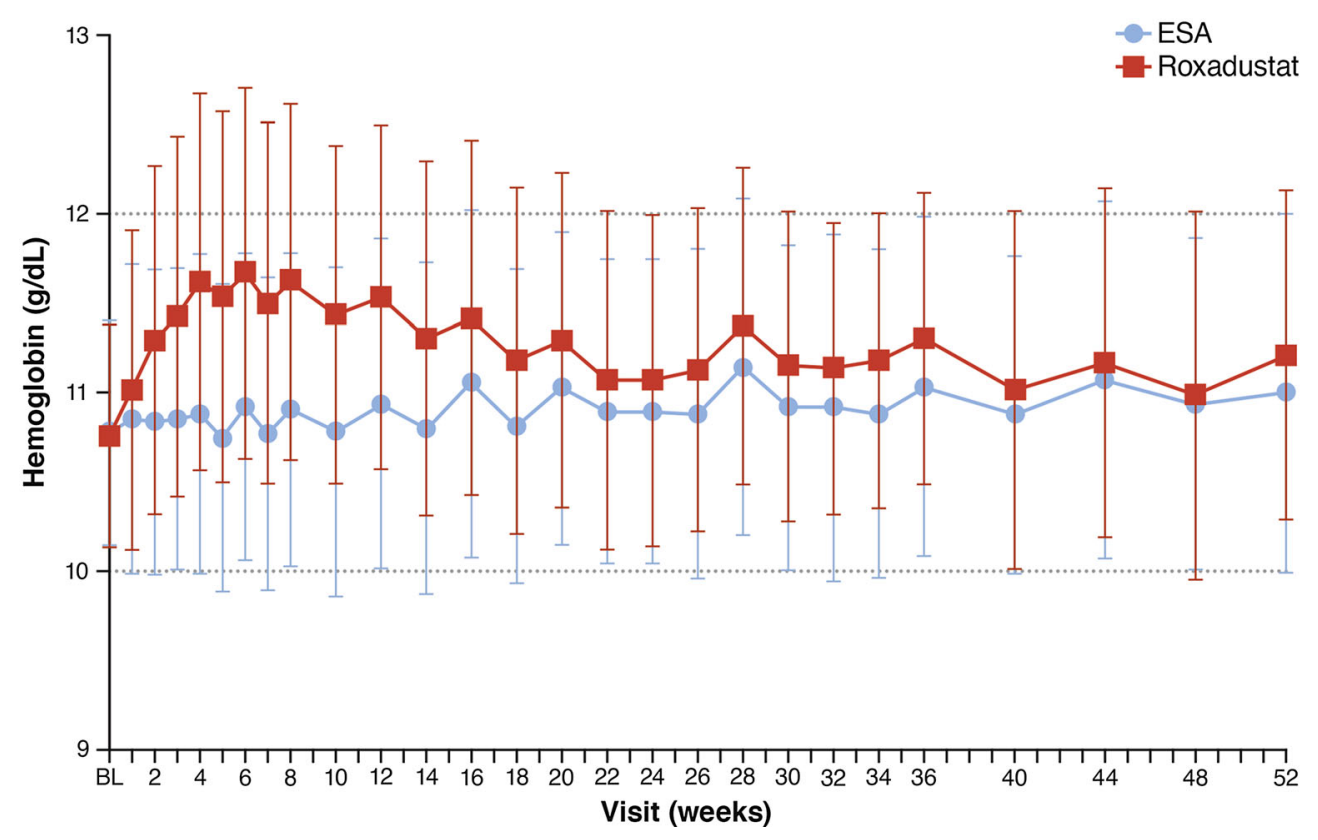

Fig. 3 Mean (SD) hemoglobin concentrations (per protocol set). $B L$ baseline, ESA erythropoiesis-stimulating agent, $S D$ standard deviation

Table 5 Statistical analysis of change in Hb levels from baseline to the average of weeks 28-52 regardless of rescue therapy ${ }^{2}$ (all randomized patients)

\begin{tabular}{lll}
\hline & Roxadustat $(\boldsymbol{n}=\mathbf{4 1 5})$ & ESA $(\boldsymbol{n}=\mathbf{4 2 1})$ \\
\hline Baseline Hb, g/dL & $10.747(0.617)$ & $10.775(0.621)$ \\
$N$ & 414 & 420 \\
Average Hb (weeks 28-52), g/dL & $11.145(0.615)$ & $10.960(0.660)$ \\
$N$ & 364 & 393 \\
Hb change from BL to average of weeks 28-52 & $0.397(0.773)$ & $0.183(0.860)$ \\
LSM (95\% CI) & $0.363(0.288,0.438)$ & $0.192(0.121,0.262)$ \\
N (imputed values) & 413 & 420 \\
LSM difference (roxadustat - ESA) & & $0.171(0.082,0.261)$ \\
Two-sided $P$-value (superiority) & & $<0.001$ \\
\hline
\end{tabular}

Data are reported as mean (standard deviation) unless otherwise indicated

$B L$ baseline, $C I$ confidence interval, ESA erythropoiesis-stimulating agent, $H b$ hemoglobin, $L S M$ least squares mean

For the ANCOVA with multiple imputation analysis, missing post-baseline values are imputed where $\mathrm{Hb}$ values averaged over 1000 simulations

${ }^{a}$ One course of rescue ESA was allowed in patients taking roxadustat if the $\mathrm{Hb}$ level had not responded adequately $(<9.0 \mathrm{~g} / \mathrm{dL})$ after at least two roxadustat dose increases in the previous 8 weeks, or if the roxadustat dose had reached the maximum limit 
Table 6 Fixed-sequence testing procedure for the key secondary endpoints

\begin{tabular}{|c|c|c|c|c|c|c|c|c|}
\hline & \multirow[t]{2}{*}{ Variable } & \multicolumn{2}{|l|}{ Result $^{a}$} & \multirow{2}{*}{$\begin{array}{l}\text { Point } \\
\text { estimate } \\
(95 \% \mathrm{CI})\end{array}$} & \multirow{2}{*}{$\begin{array}{l}\text { Statistics } \\
\text { (units) }\end{array}$} & \multirow[t]{2}{*}{$P$ value $^{b}$} & \multirow{2}{*}{$\begin{array}{l}\text { Test } \\
\text { (analysis set) }\end{array}$} & \multirow{2}{*}{$\begin{array}{l}\text { Non- } \\
\text { inferiority } \\
\text { margin }\end{array}$} \\
\hline & & Roxadustat & ESA & & & & & \\
\hline 1 & $\begin{array}{l}\text { Proportions of } \\
\text { responders without } \\
\text { having received } \\
\text { rescue therapy } \\
\text { within } 6 \text { weeks } \\
\text { prior to and during } \\
\text { weeks } 28-36\end{array}$ & $84.2 \%$ & $82.4 \%$ & $\begin{array}{l}2.3 \%(-2.9, \\
7.6)\end{array}$ & $\begin{array}{r}\text { Difference of } \\
\text { proportions }\end{array}$ & NA & $\begin{array}{l}\text { Non- } \\
\text { inferiority }^{\mathrm{c}} \\
\text { (PPS) }\end{array}$ & $-15 \%$ \\
\hline 2 & $\begin{array}{l}\text { Change in LDL } \\
\text { cholesterol from } \\
\text { baseline to the } \\
\text { average LDL } \\
\text { cholesterol (mmol/ } \\
\text { L) in weeks } 12-28\end{array}$ & -0.459 & -0.082 & $\begin{array}{l}-0.377 \\
\quad(-0.451 \\
-0.304)\end{array}$ & $\begin{array}{l}\text { Difference of } \\
\text { LSM } \\
(\mathrm{mmol} / \mathrm{L})\end{array}$ & $<0.001$ & $\begin{array}{l}\text { Superiority } \\
\text { (FAS) }\end{array}$ & NA \\
\hline 3 & $\begin{array}{l}\text { Mean monthly IV } \\
\text { iron use (mg) from } \\
\text { day } 1 \text { to week } 36\end{array}$ & 21.6 & 53.5 & $\begin{array}{l}-31.9 \\
\quad(-41.4 \\
-22.4)\end{array}$ & $\begin{array}{c}\text { Difference of } \\
\text { LSM (mg) }\end{array}$ & $<0.001$ & $\begin{array}{l}\text { Superiority } \\
\text { (FAS) }\end{array}$ & NA \\
\hline 4 & $\begin{array}{l}\text { Change in SF-36 PF } \\
\text { sub-score from } \\
\text { baseline to the } \\
\text { average of } \\
\text { weeks 12-28 }\end{array}$ & 0.050 & -0.155 & $\begin{array}{l}0.205 \\
(-0.649 \\
1.059)\end{array}$ & $\begin{array}{l}\text { Difference of } \\
\text { LSM }\end{array}$ & NA & $\begin{array}{l}\text { Non- } \\
\text { inferiority }^{\mathrm{c}} \\
\text { (PPS) }\end{array}$ & -3 \\
\hline 5 & $\begin{array}{l}\text { Change in SF-36 VT } \\
\text { sub-score from } \\
\text { baseline to the } \\
\text { average of } \\
\text { weeks 12-28 }\end{array}$ & 0.460 & -0.396 & $\begin{array}{l}0.856 \\
\quad(-0.115 \\
1.828)\end{array}$ & $\begin{array}{l}\text { Difference of } \\
\text { LSM }\end{array}$ & NA & $\begin{array}{l}\text { Non- } \\
\text { inferiority }^{\mathrm{c}} \\
(\mathrm{PPS})\end{array}$ & -3 \\
\hline 6 & $\begin{array}{l}\text { Change in MAP } \\
\text { (mmHg) from } \\
\text { baseline to } \\
\text { weeks 20-28 }\end{array}$ & -0.969 & -0.120 & $\begin{array}{c}-0.849 \\
(-1.971 \\
0.273)\end{array}$ & $\begin{array}{l}\text { Difference of } \\
\text { LSM } \\
(\mathrm{mmHg})\end{array}$ & NA & $\begin{array}{l}\text { Non- } \\
\text { inferiority }^{\mathrm{d}} \\
\text { (PPS) }\end{array}$ & +1 \\
\hline 7 & $\begin{array}{l}\text { Time to increase in } \\
\text { blood pressure } \\
\text { between weeks } 1 \\
\text { and } 36\end{array}$ & 32.2 & 35.7 & $\begin{array}{c}0.924 \\
(0.669 \\
1.276)\end{array}$ & Hazard ratio & NA & $\begin{array}{l}\text { Non- } \\
\text { inferiority }^{\mathrm{d}} \\
\text { (PPS) }\end{array}$ & +1.3 \\
\hline 8 & $\begin{array}{l}\text { Change in MAP } \\
\text { (mmHg) from } \\
\text { baseline to } \\
\text { weeks } 20-28\end{array}$ & -0.739 & -0.160 & $\begin{array}{c}-0.579 \\
(-1.694 \\
0.536)\end{array}$ & $\begin{array}{l}\text { Difference of } \\
\text { LSM } \\
(\mathrm{mmHg})\end{array}$ & 0.308 & $\begin{array}{l}\text { Superiority } \\
\text { (FAS) }\end{array}$ & NA \\
\hline
\end{tabular}


Table 6 continued

\begin{tabular}{|c|c|c|c|c|c|c|c|c|}
\hline & \multirow[t]{2}{*}{ Variable } & \multicolumn{2}{|l|}{ Result $^{a}$} & \multirow{2}{*}{$\begin{array}{l}\text { Point } \\
\text { estimate } \\
\text { (95\% CI) }\end{array}$} & \multirow{2}{*}{$\begin{array}{l}\text { Statistics } \\
\text { (units) }\end{array}$} & \multirow[t]{2}{*}{$P$ value $^{\mathrm{b}}$} & \multirow{2}{*}{$\begin{array}{l}\text { Test } \\
\text { (analysis set) }\end{array}$} & \multirow{2}{*}{$\begin{array}{l}\text { Non- } \\
\text { inferiority } \\
\text { margin }\end{array}$} \\
\hline & & Roxadustat & ESA & & & & & \\
\hline 9 & $\begin{array}{l}\text { Time to increase in } \\
\text { blood pressure } \\
\text { between weeks } 1 \\
\text { and } 36\end{array}$ & 32.8 & 37.2 & $\begin{array}{l}0.915 \\
(0.668 \\
1.254)\end{array}$ & Hazard ratio & 0.582 & $\begin{array}{l}\text { Superiority } \\
\text { (FAS) }\end{array}$ & NA \\
\hline
\end{tabular}

All the analyses compared roxadustat versus ESA

$C I$ confidence interval, ESA erythropoiesis-stimulating agent, $F A S$ full analysis set, $I V$ intravenous, $L D L$ low-density lipoprotein, $L S M$ least squares mean, $M A P$ mean arterial pressure, $N A$ not applicable, $P P S$ per protocol set, $S D$ standard deviation, $S F-36$ PF Short Form-36 health survey Physical Functioning, SF-36 VT Short Form-36 health survey Vitality

${ }^{a}$ Incidence rates were compared for time to event variables and LSM were compared for all other variables

${ }^{\mathrm{b}}$ Values are presented for superiority test only

${ }^{c}$ Non-inferiority was concluded if the lower bound of the $95 \%$ CI of the difference between groups was above the noninferiority margin

${ }^{d}$ Non-inferiority was concluded if the upper bound of the 95\% CI of the difference between groups was below the noninferiority margin

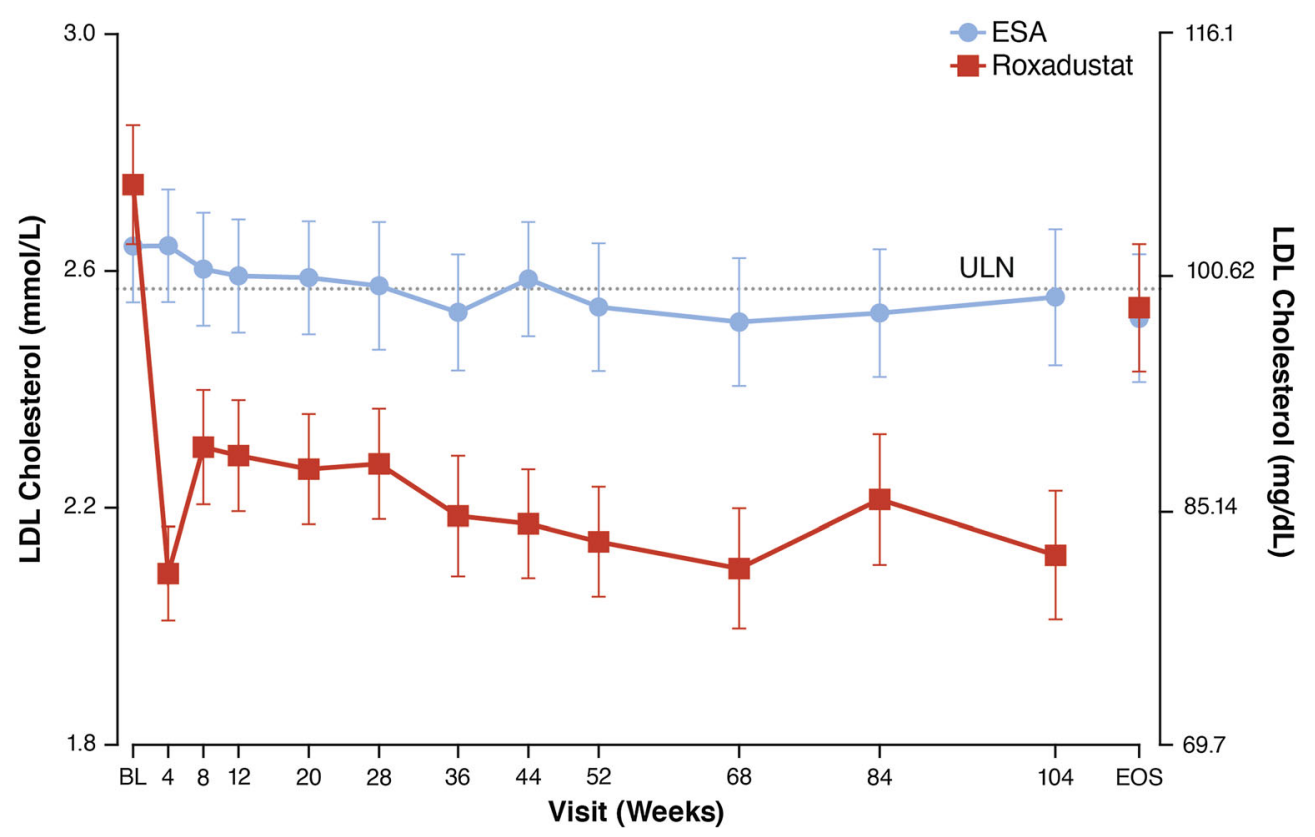

Fig. 4 Mean (SD) low-density lipoprotein cholesterol by visit (full analysis set). ESA erythropoiesis-stimulating agent, LDL low-density lipoprotein

Throughout the study, the levels of hepcidin and ferritin were lower in the roxadustat versus the ESA group, whereas TSAT levels were comparable (Supplemental Results and Supplemental Figs. S6, S7, and S8). The mean (SD) change from baseline to week 52 in serum iron was $-0.30(7.43) \mu \mathrm{mol} / \mathrm{L}$ and $-1.23(6.31) \mu \mathrm{mol} / \mathrm{L}$ in roxadustat- and ESA-treated patients, respectively. The mean (SD) change from baseline to week 52 in total iron binding capacity (TIBC) 
Table 7 Overview of treatment-emergent adverse events (safety analysis set) ${ }^{a}$

\begin{tabular}{lcc}
\hline & $\begin{array}{l}\text { Roxadustat } \\
(\boldsymbol{n}=\mathbf{4 1 4})\end{array}$ & $\begin{array}{l}\text { ESA } \\
(\boldsymbol{n}=\mathbf{4 2 0})\end{array}$ \\
\hline TEAE & $359(86.7)$ & $361(86.0)$ \\
Serious TEAE & $210(50.7)$ & $189(45.0)$ \\
$\begin{array}{l}\text { TEAE leading to } \\
\text { discontinuation of study } \\
\text { drug b }\end{array}$ & $35(8.5)$ & $16(3.8)$ \\
$\begin{array}{l}\text { TEAE leading to death } \\
\begin{array}{l}\text { Death during the safety } \\
\text { emergent period }\end{array}\end{array}$ & $67(16.2)$ & $55(13.1)$ \\
\hline
\end{tabular}

Data are reported as $n$ (\%)

$E S A$ erythropoiesis-stimulating agent, TEAE treatmentemergent adverse event

a Period from the first study drug administration up to 28 days after the last dose

b Includes patients with at least one TEAE who permanently discontinued the study drug

was $9.98(8.84) \mu \mathrm{mol} / \mathrm{L}$ and $2.74(6.39) \mu \mathrm{mol} / \mathrm{L}$ in roxadustat- and ESA-treated patients, respectively.

\section{Safety}

The proportion of patients who reported TEAEs was similar in the roxadustat group $(86.7 \%)$ and ESA group (86.0\%). Serious TEAEs were reported in $50.7 \%$ and $45.0 \%$ of patients in the roxadustat and ESA groups, respectively. The proportion of patients reporting TEAEs leading to discontinuation of study drug was higher in the roxadustat group than in the ESA group $(8.5 \%$ vs $3.8 \%$ ). Death due to any cause after the first study drug administration was reported in $18.8 \%$ and $14.0 \%$ of patients in the roxadustat group and the ESA group, respectively. TEAEs leading to death were reported in $16.2 \%$ and $13.1 \%$ of patients in the roxadustat group and the ESA group, respectively (Table 7). Cumulative number of deaths over time was different between treatment groups after approximately 6 months of treatment (Supplemental Fig. S9). Estimated Kaplan-Meier risks of death comparing roxadustat and ESAs were 3.3 (95\% CI 1.5, 5.0) for roxadustat vs 2.4 (95\% CI 0.9 , 3.9) for ESA at 6 months; 8.3 (95\% CI 5.5, 11.1) vs 5.4 (95\% CI $3.2,7.7)$ at 12 months; 13.1 (95\% CI 9.6, 16.6) vs 6.8 (95\% CI $4.3,9.2)$ at 18 months. Cardiac disorders were the most common TEAEs leading to death in both the roxadustat group $(5.8 \%)$ and the ESA group (5.5\%) (Supplementary Table S9).

Within the ESA arm, mortality differed according to treatment prior to the study. In the subgroup of patients pretreated with DA, the overall incidence of death was similar in the roxadustat and DA subgroups $(n=21 / 158$ [13.3\%] vs $n=22 / 163$ [13.5\%]). In the subgroup of patients pretreated with epoetin alfa, more fatal events occurred in the roxadustat group than in the epoetin alfa subgroup $(n=57 / 256$ [22.3\%] vs $n=37 / 257$ [14.4\%]). Further exploration of this finding is provided in the discussion and in the additional subgroup analyses of the effect of baseline factors on mortality reported in Supplemental Table S10.

Some of the most common TEAEs in both treatment groups included hypertension, arteriovenous fistula thrombosis, headache, and diarrhea. The TEAEs of arteriovenous fistula thrombosis (12.1\% vs $7.4 \%)$ and nausea (7.0\% vs $1.9 \%)$ were reported more frequently in the roxadustat group than in the ESA group, whereas iron deficiency $(7.2 \%$ vs $12.1 \%)$ and muscle spasm $(3.6 \%$ vs $7.9 \%)$ were reported more frequently in the ESA group (Table 8).

\section{DISCUSSION}

This study evaluated the efficacy and safety of roxadustat compared with ESAs (epoetin alfa and DA) in the maintenance treatment of anemia in a European population on dialysis who were previously on stable doses of an ESA. Roxadustat was non-inferior to ESAs for the efficacy endpoints of change in $\mathrm{Hb}$ levels and proportion of responders. Furthermore, roxadustat was non-inferior to ESAs for the change in $\mathrm{Hb}$ levels from baseline to weeks 28-52 (regardless of rescue therapy), and superior for the ability to lower LDL cholesterol and to reduce the use of IV iron. These findings confirm and 
Table 8 Event rates of common (at least 5\% in any treatment group) treatment-emergent adverse events (safety analysis set)

\begin{tabular}{|c|c|c|c|c|}
\hline \multirow[t]{2}{*}{ MedDRA v20.0 preferred term } & \multicolumn{2}{|c|}{ Roxadustat $(n=414 ;$ PEY $=662.2)$} & \multicolumn{2}{|c|}{ ESA $(n=420 ;$ PEY $=752.2)$} \\
\hline & $\overline{N(\%)}$ & $\overline{\# E(\text { Event rate/100 PEY) }}{ }^{\mathrm{a}}$ & $N(\%)$ & $\# E$ (Event rate/100 PEY) \\
\hline Hypertension & $74(17.9)$ & $111(16.8)$ & $79(18.8)$ & $121(16.1)$ \\
\hline Arteriovenous fistula thrombosis & $50(12.1)$ & $71(10.7)$ & $31(7.4)$ & $39(5.2)$ \\
\hline Headache & $36(8.7)$ & $41(6.2)$ & $29(6.9)$ & $39(5.2)$ \\
\hline Diarrhea & $35(8.5)$ & $53(8.0)$ & $35(8.3)$ & $64(8.5)$ \\
\hline Bronchitis & $33(8.0)$ & $43(6.5)$ & $29(6.9)$ & $37(4.9)$ \\
\hline Hypotension & $33(8.0)$ & $45(6.8)$ & $27(6.4)$ & $43(5.7)$ \\
\hline Iron deficiency & $30(7.2)$ & $39(5.9)$ & $51(12.1)$ & $64(8.5)$ \\
\hline Nausea & $29(7.0)$ & $31(4.7)$ & $8(1.9)$ & $10(1.3)$ \\
\hline Viral upper respiratory tract infection & $29(7.0)$ & $62(9.4)$ & $39(9.3)$ & $68(9.0)$ \\
\hline Arteriovenous fistula site complication & $23(5.6)$ & $38(5.7)$ & $21(5.0)$ & $31(4.1)$ \\
\hline Pneumonia & $23(5.6)$ & $27(4.1)$ & $27(6.4)$ & $30(4.0)$ \\
\hline Hyperparathyroidism secondary & $22(5.3)$ & $24(3.6)$ & $16(3.8)$ & $17(2.3)$ \\
\hline Anemia & $21(5.1)$ & $27(4.1)$ & $16(3.8)$ & $21(2.8)$ \\
\hline Atrial fibrillation & $20(4.8)$ & $27(4.1)$ & $25(6.0)$ & $27(3.6)$ \\
\hline Muscle spasms & $15(3.6)$ & $21(3.2)$ & $33(7.9)$ & $48(6.4)$ \\
\hline Upper respiratory tract infection & $14(3.4)$ & $20(3.0)$ & $22(5.2)$ & $30(4.0)$ \\
\hline
\end{tabular}

\#E number of events, ESA erythropoiesis-stimulating agent, $P E Y$ patient exposure years

a Event rate/100 PEY: (number of events) $\times 100$ divided by PEY during the safety emergent period

extend those from previous studies that examined roxadustat and ESAs in patients with DD CKD [11, 16-19].

In line with previous studies [11, 17-19], roxadustat was superior to ESAs in its ability to lower LDL cholesterol levels, with a greater decrease in mean LDL cholesterol from baseline to the average of weeks $12-28$ observed in the roxadustat group versus the ESA group. One potential mechanism hypothesized to contribute to cholesterol-lowering effect of roxadustat is a proposed hypoxia-induced induction of an insulin-induced gene, which can stimulate the degradation of hydroxymethylglutaryl coenzyme A reductase [20]. The physiological and clinical effects of reduced
LDL cholesterol over time in this population warrant further investigation.

Superiority of roxadustat to ESA was demonstrated for the use of IV iron for the proportion of patients requiring IV iron from baseline to week 36 and mean monthly dose. In both treatment groups, the baseline levels of serum ferritin were higher than those recommended by the KDIGO guideline. A more rapid decrease in ferritin levels was observed in the roxadustat group, which approached the upper limits of KDIGO recommendations, whereas the levels remained above $1400 \mathrm{ng} / \mathrm{mL}$ in the ESA group. Additionally, in line with previous studies [11-13], we observed a relative reduction in hepcidin levels in the roxadustat group 
versus the ESA group. Since increased hepcidin levels have been associated with inflammation and functional iron deficiency [21], reduced hepcidin may result in improved iron absorption and mobilization.

The effect of ESA treatment in improving the HRQoL of DD and NDD patients has been previously reported using different assessment tools $[9,22]$. In this study, HRQoL measures were improved with roxadustat and ESA. Though a greater proportion of patients in the roxadustat group reported an improvement in the PGIC scale, changes in other measurements, such as the SF-36, FACT-An, and EQ-5D-5L VAS scores, were comparable between treatment groups.

Overall, the type of adverse events reported in this study for a mostly European population are similar to those reported in previous studies $[11,13,17-19,23]$. The incidences of serious TEAEs and TEAEs leading to treatment discontinuation as well as overall study withdrawal were higher in the roxadustat group compared with the ESA group. This is not unexpected considering this was an open-label trial and because of the high rate of background events common in this population. Furthermore, while these differences may be reproducible in other studies, these differences may also be incidental findings or may be due to this study's comparison of a standard of care therapy (ESA) versus a new therapy (roxadustat). It seems logical that patients might be interested in returning to a previous therapy if they experience an adverse event on an experimental therapy. Among the most common serious TEAEs, none of the events differed in rate by of 2.0 or more (except for arteriovenous fistula thrombosis) per 100 PEY.

While this study was not powered to detect non-inferiority in terms of cardiovascular safety and mortality outcomes, an apparent difference in deaths between the roxadustat and ESA groups was observed during the safety emergent period (15.5\% vs $12.1 \%$ ) and was driven by the comparison of roxadustat versus epoetin alfa rather than roxadustat versus DA with comparable event rates between the DA and epoetin alfa arms and in the roxadustat arm in the DA subgroup. While various baseline factors showed an association with mortality in general (primarily age, cardiovascular history, and diabetes), none of the baseline factors included in the Cox regression model as co-variates (age; age cutoffs 65 years/75 years; prior ESA type; prior ESA dose; history of cardiovascular, cerebrovascular, or thromboembolic diseases; history of diabetes; use of ACE/ARB medication; and baseline LDL cholesterol and dialysis vintage) had an impact on the estimated treatment effect (i.e., mortality risk with roxadustat vs ESA).

Nevertheless, in addition to the observed imbalances in demographics between the treatment groups, clinical factors and variations in practice patterns not captured in the study may have been affected by other conditions associated with cardiovascular risk in dialysis patients. For example, in the subgroup of patients who had been pre-treated with epoetin alfa, the mean dialysis vintage in the patients on roxadustat at baseline (4.63 years) was roughly $26 \%$ longer than in the ESA group (3.67 years). As patients on dialysis who survive more than 2 years already have an increased risk of mortality, this baseline difference may have affected outcomes, such as mortality, in all subgroups. Furthermore, this difference also suggests that other baseline differences affecting mortality could have been present, supporting the traditional approach of basing safety assessments on the largest available patient population.

In addition, the ESA conversion design in this study complicates interpretation of safety and mortality data. Patients enrolled in the PYRENEES study were pre-treated with stable doses of ESA and generally had maintained $\mathrm{Hb}$ levels within, or close to, the target range. Upon randomization and study treatment initiation, patients randomized to ESA continued the same type of treatment, whereas those randomized to roxadustat were switched to a new treatment modality with a different route of administration and a different mechanism of action. Consequently, one-sided fluctuations in anemia management (upon switching over to treatment with roxadustat) are conceivable. This provides a plausible basis for a potentially design-related bias that may 
have impacted the difference in outcomes, including mortality, between the ESA- and roxadustat-treated patients; however, it does not explain the differences in both subgroups or the difference in mortality observed after the first 6 months of treatment.

The PYRENEES study was designed as part of a clinical development program for roxadustat, with the intention to pool multiple studies in order to provide sufficient power for safety assessment; the safety findings of a given single study may randomly differ from that of the aggregate. In contrast with this study in ESApre-treated patients on dialysis for at least 4 months converted to roxadustat, a pooled analyses of a dialysis patient cohort, regardless of ESA pretreatment and time on dialysis collected across multiple phase 3 studies, showed a similar safety profile between roxadustat and ESA (epoetin alfa and DA), with similar rates of adjudicated death and major adverse cardiovascular events (MACE) and MACE plus hospitalization for congestive heart failure or unstable angina (MACE+) events for roxadustat and epoetin alfa $[24,25]$.

Other limitations besides the ESA conversion design bias should be considered when evaluating the results of this study. Firstly, imbalances between groups (e.g., baseline comorbidities) exist, as randomization can never eliminate all imbalances, and these imbalances could have affected outcomes and/ or confounded the current comparison. Unmeasured confounding variables and conversion/design-related confounding should be considered alongside roxadustat, and additional investigation is warranted to explain the study results. The use of an open-label design could increase the risk of bias; however, a doubleblind study design was not feasible considering the use of two different active comparators with distinct dosing specifications and the need for dose adjustments throughout the study [26]. Interpretation of the imbalances in the number of deaths between treatment groups is difficult because this study was underpowered for this outcome and may have been confounded by the fact that ESAs were used for anemia treatment in patients who prematurely discontinued study treatment after the last dose of study drug until the preplanned 2-year closure of observation. Another limitation is that the population was almost entirely from Central and Eastern Europe, which may limit the generalizability of the findings because of differences in treatment practices across regions.

\section{CONCLUSIONS}

Overall, this study shows that roxadustat is noninferior to ESAs (epoetin alfa and DA) in maintaining $\mathrm{Hb}$ levels within 10.0-12.0 g/dL for up to 104 weeks in dialyzed patients with anemia of CKD who were previously treated with ESAs. The effects of roxadustat and ESAs on HRQoL were comparable. In general, the types of TEAEs observed in this study are in line with previous studies.

\section{ACKNOWLEDGEMENTS}

We thank the participants of the study.

Funding. This study and publication fees were funded by Astellas Pharma, Inc.

Medical Writing, Editorial, and Other Assistance. Medical writing/editorial support was provided by Rosalba Satta, PhD, Patrick Tucker, PhD, Drayton Hammond, PharmD, and Elizabeth Hermans, PhD, from Peloton Advantage, LLC, an OPEN Health company, Parsippany, NJ, and funded by the study sponsor.

Authorship. All named authors meet the International Committee of Medical Journal Editors (ICMJE) criteria for authorship for this article, take responsibility for the integrity of the work as a whole, and have given their approval for this version to be published.

Author Contributions. Conception and design: MR; Acquisition of data: BC, MR, CE, JB, WS, MS; Analysis and interpretation of the data: MR, CE, WS, UV; Drafting and critical revision of the article for important intellectual content: $\mathrm{BC}, \mathrm{MR}, \mathrm{CE}, \mathrm{JB}, \mathrm{WS}, \mathrm{MS}, \mathrm{UV}$. 
Prior Presentation. Results from this study were presented at the American Society of Nephrology Kidney Week on November 9, 2019 (Abstract: SA-PO225).

Disclosures. U. Valluri is an employee of Astellas Pharma, Inc. M. Reusch is an employee of Astellas Pharma Europe B.V. J. Barratt, B. Csiky, C. Esposito, M. Schömig, and W. Sulowicz have nothing to disclose.

Compliance with Ethics Guidelines. This study was conducted in accordance with the ethical principles of the Declaration of Helsinki, Good Clinical Practice, the International Committee on Harmonisation guidelines, and applicable laws and regulations. The protocol was approved by each institutional review board and all subjects provided written informed consent.

Data Availability. Researchers may request access to anonymized participant level data, trial level data and protocols from Astellas sponsored clinical trials at www. clinicalstudydatarequest.com. For the Astellas criteria on data sharing see: https:// clinicalstudydatarequest.com/Study-Sponsors/ Study-Sponsors-Astellas.aspx.

Open Access. This article is licensed under a Creative Commons Attribution-NonCommercial 4.0 International License, which permits any non-commercial use, sharing, adaptation, distribution and reproduction in any medium or format, as long as you give appropriate credit to the original author(s) and the source, provide a link to the Creative Commons licence, and indicate if changes were made. The images or other third party material in this article are included in the article's Creative Commons licence, unless indicated otherwise in a credit line to the material. If material is not included in the article's Creative Commons licence and your intended use is not permitted by statutory regulation or exceeds the permitted use, you will need to obtain permission directly from the copyright holder. To view a copy of this licence, visit http:// creativecommons.org/licenses/by-nc/4.0/.

\section{REFERENCES}

1. Locatelli F, Fishbane S, Block GA, Macdougall IC. Targeting hypoxia-inducible factors for the treatment of anemia in chronic kidney disease patients. Am J Nephrol. 2017;45(3):187-99.

2. Babitt JL, Lin HY. Mechanisms of anemia in CKD. J Am Soc Nephrol. 2012;23(10):1631-4.

3. Sibbel SP, Koro CE, Brunelli SM, Cobitz AR. Characterization of chronic and acute ESA hyporesponse: a retrospective cohort study of hemodialysis patients. BMC Nephrol. 2015;16:144.

4. Luo J, Jensen DE, Maroni BJ, Brunelli SM. Spectrum and burden of erythropoiesis-stimulating agent hyporesponsiveness among contemporary hemodialysis patients. Am J Kidney Dis. 2016;68(5): 763-71.

5. Johnson DW, Pollock CA, Macdougall IC. Erythropoiesis-stimulating agent hyporesponsiveness. Nephrology (Carlton). 2007;12(4):321-30.

6. Ingrasciotta $\mathrm{Y}$, Giorgianni $\mathrm{F}$, Marciano $\mathrm{I}$, et al. Comparative effectiveness of biosimilar, reference product and other erythropoiesis-stimulating agents (ESAs) still covered by patent in chronic kidney disease and cancer patients: an Italian population-based study. PLOS ONE. 2016;11(5): e0155805.

7. Pfeffer MA, Burdmann EA, Chen CY, et al. A trial of darbepoetin alfa in type 2 diabetes and chronic kidney disease. N Engl J Med. 2009;361(21): 2019-32.

8. Besarab A, Bolton WK, Browne JK, et al. The effects of normal as compared with low hematocrit values in patients with cardiac disease who are receiving hemodialysis and epoetin. $\mathrm{N}$ Engl J Med. 1998;339(9):584-90.

9. Singh AK, Szczech L, Tang KL, et al. Correction of anemia with epoetin alfa in chronic kidney disease. N Engl J Med. 2006;355(20):2085-98.

10. Gupta N, Wish JB. Hypoxia-inducible factor prolyl hydroxylase inhibitors: a potential new treatment for anemia in patients with CKD. Am J Kidney Dis. 2017;69(6):815-26.

11. Chen N, Hao C, Liu BC, et al. Roxadustat treatment for anemia in patients undergoing long-term dialysis. N Engl J Med. 2019;381(11):1011-22.

12. Akizawa T, Otsuka T, Reusch M, Ueno M. Intermittent oral dosing of roxadustat in peritoneal dialysis chronic kidney disease patients with 
anemia: a randomized, phase 3 , multicenter, openlabel study. Ther Apher Dial. 2020;24(2):115-25.

13. Akizawa T, Ueno M, Shiga T, Reusch M. Oral roxadustat three times weekly in ESA-naive and ESAconverted patients with anemia of chronic kidney disease on hemodialysis: results from two phase 3 studies. Ther Apher Dial. 2020;24(6):628-41.

14. Barratt J, Andric B, Tataradze A, et al. Roxadustat for the treatment of anaemia in chronic kidney disease patients not on dialysis: a phase 3 , randomised, open-label, active-controlled study (DOLOMITES). Nephrol Dial Transp. 2021;36(9):1616.

15. Chen N, Hao C, Peng X, et al. Roxadustat for anemia in patients with kidney disease not receiving dialysis. N Engl J Med. 2019;381(11):1001-10.

16. Akizawa T, Iwasaki M, Yamaguchi Y, Majikawa Y, Reusch M. Phase 3, randomized, double-blind, active-comparator (darbepoetin alfa) study of oral roxadustat in CKD patients with anemia on hemodialysis in Japan. J Am Soc Nephrol. 2020;31(7):1628-39.

17. Provenzano R, Shutov E, Eremeeva L, et al. Roxadustat for anemia in patients with end-stage renal disease incident to dialysis. Nephrol Dial Transp. 2021;36(9):1717.

18. Fishbane S, Pollock CA, El-Shahawy $M$, et al. Roxadustat versus epoetin alfa for treating anemia in patients with chronic kidney disease on dialysis: results from the randomized phase 3 ROCKIES study. J Am Soc Nephrol. 2021;32(3):737.

19. Charytan C, Manllo-Karim R, Martin ER, Steer D, Bernardo M, Dua SL, Moustafa MA, Saha G, Bradley C, Eyassu M, Leong R, Saikali KG, Liu C, Szczech L, $\mathrm{Yu} \mathrm{KP}$. A randomized trial of roxadustat in anemia of kidney failure: SIERRAS study. Kidney Int Rep. 2021;6(7):1829-39.
20. Hwang S, Nguyen AD, Jo Y, Engelking LJ, Brugarolas J, DeBose-Boyd RA. Hypoxia-inducible factor $1 \alpha$ activates insulin-induced gene 2 (Insig-2) transcription for degradation of 3-hydroxy-3-methylglutaryl (HMG)-CoA reductase in the liver. J Biol Chem. 2017;292(22):9382-93.

21. Ganz T. Hepcidin, a key regulator of iron metabolism and mediator of anemia of inflammation. Blood. 2003;102(3):783-8.

22. Jones M, Ibels L, Schenkel B, Zagari M. Impact of epoetin alfa on clinical end points in patients with chronic renal failure: a meta-analysis. Kidney Int. 2004;65(3):757-67.

23. Poole L, Saha G, Bradley C, WHO. Himalayas: a phase 3, randomized, open-label, active-controlled study of the efficacy and safety of roxadustat in the treatment of anemia in incident-dialysis patients. Washington: American Society of Nephrology Kidney Week; 2019.

24. Fishbane S, El-Shahawy MA, Pecoits-Filho R, Van BP, Houser MT, Frison L, Little DJ, Guzman NJ, Pergola PE. Roxadustat for treating anemia in patients with CKD not on dialysis: Results from a randomized phase 3 study. J Am Soc Nephrol. 2021;32(3):737-55.

25. Barratt J, Sulowicz W, Schömig M, et al. Efficacy and cardiovascular safety of roxadustat in dialysis-dependent chronic kidney disease: pooled analysis of four phase 3 studies. Adv Ther. 2021. (In preparation).

26. Wilhelm-Leen ER, Winkelmayer WC. Mortality risk of darbepoetin alfa versus epoetin alfa in patients with CKD: systematic review and meta-analysis. Am J Kidney Dis. 2015;66(1):69-74. 